\title{
Downregulation of CXCR4 by SDF-KDEL in SBC-5 cells inhibits their migration in vitro and organ metastasis in vivo
}

\author{
NINGQIANG MA ${ }^{1 *}$, HAILIN PANG $^{1 *}$, WEIWEI SHEN $^{1}$, FENG ZHANG $^{1}$, ZAOXUN CUI $^{1}$, \\ JUNYAN WANG $^{2}$, JIANLIN WANG ${ }^{3}$, LILI LIU ${ }^{1}$ and HELONG ZHANG ${ }^{1}$ \\ Departments of ${ }^{1}$ Oncology and ${ }^{2}$ Nuclear Medicine, Tangdu Hospital, The Fourth Military Medical University, \\ Xi'an, Shaanxi $710038 ;{ }^{3}$ Department of Hepatobiliary Surgery, Xijing Hospital, \\ The Fourth Military Medical University, Xi'an, Shaanxi 710032, P.R. China
}

Received August 27, 2014; Accepted December 1, 2014

DOI: $10.3892 /$ ijmm.2014.2033

\begin{abstract}
Metastasis is the principal cause of morbidity and mortality in cancer patients. The master genes that govern organ-selective metastasis remain elusive. We compared the expression levels of C-X-C chemokine receptor type 4 (CXCR4) in the human small cell lung cancer (SCLC) cells, SBC-5 and SBC-3, by flow cytometric analysis and found that CXCR4 was expressed at markedly higher levels in the SBC-5 cells which can produce multiple organ metastasis, particularly bone metastasis compared to the SBC-3 cells. Stromal-derivedfactor-1 (SDF-1)-CXCR4 has been shown to regulate cell migration and metastasis in a various types of cancer; however, the roles of SDF-1-CXCR4 in the organ-selective metastasis of SCLC in vivo remain to be elucidated. Thus, in this study, we constructed a phenotype of SBC-5 cells in which CXCR4 was knocked out using the intrakine strategy and found that the downregulation of CXCR4 inhibited cell migration and invasion, but did not affect cell proliferation or apoptosis in vitro. In in vivo experiments, the knockout of CXCR4 suppressed the development of metastastic lesions in the lungs, liver and bone, but did not decrease metastasis to the kidneys. Our data demonstrate that CXCR4 is a candidate gene involved in the development of metastastic lesions in specific organs, such as the lungs, bone and liver, which can secrete high concentrations of SDF-1, the sole ligand of CXCR4. Thus, CXCR4 may prove to be a promising target for the prevention and effective treatment of metastastic lesions due to SCLC.
\end{abstract}

Correspondence to: Professor Lili Liu or Professor Helong Zhang, Department of Oncology, Tangdu Hospital, The Fourth Military Medical University, 1 Xinsi Road, Xi'an, Shaanxi 710038, P.R. China E-mail: lily123fmmu@163.com

E-mail: cnxazhl@163.com

*Contributed equally

Key words: SDF-1-CXCR4, intrakine, metastasis, small-cell lung cancer

\section{Introduction}

Metastasis is the principal cause of morbidity and mortality in cancer patients. It occurs during the late stages of tumor progression and is an exceedingly complex event (1). Of note, certain types of cancer show a predilection to metastasis to specific organs (2). In 1889, Stephen Paget set forth the 'seed and soil' hypothesis of metastasis, to explain the non-random pattern of metastasis (3). The theory states that metastasis is dependent on the cross-talk between selected cancer cells (the 'seeds') and specific organ microenvironments (the 'soil'), and this theory still holds forth today. However, the precise molecular mechanisms, regulatory circuits and master metastasis-associated genes that govern these fatal changes remain elusive (4). Small cell lung cancer (SCLC) accounts for $15-20 \%$ of lung cancer cases and presents an aggressive clinical behavior characterized by rapid growth and spread to distant organs (5). Previously, Miki et al (6) examined the ability of various lung cancer cell lines to generate multiple organ metastasis, particularly bone metastasis by intravenously injecting the cancer cells into natural killer (NK) cell-depleted SCID mice; they found that the human the SCLC cell line, SBC-5 was the only one to generate bone metastasis; bone metastasis was not generated by the SBC-3 cells (the SBC-3 cell line was originally established from the bone marrow aspirate of a 24-year-old male patient with SCLC). Although the SBC-5 and SBC-3 cells have a similar genetic background, they differ in their potential to generate bone metastasis. In this study, we compared the surface expression of C-X-C chemokine receptor type 4 (CXCR4) proteins in SBC-5 and SBC-3 cells by flow cytometric analysis, and the results demonstrated that $\mathrm{CXCR} 4$ protein expression was markedly higher in the SBC-5 cells compared with the SBC- 3 cells. It has previously been demonstrated that CXCR4 and stromalderived-factor-1 (SDF-1) regulate migration and metastasis in certain types of cancer (7); however, the roles of SDF-1-CXCR4 in the organ-selective metastasis of SCLC remain to be elucidated.

The term intrakine (intracellular chemokine) refers to the strategy used to genetically silence a certain (cytokine) receptor. This is achieved by the fusion of the chemokine gene with an endoplasmic reticulum (ER) retention signal (KDEL), termed 'intrakine', which can bind to the newly synthesized 
chemokine receptor molecules within the cell and block their surface expression. This method was originally proposed as gene therapy for AIDS (8-10). Thereafter, it has been used as a tool for the functional analysis of chemokines and their receptors in vitro and in vivo (11).

In the present study, we knocked down CXCR4 expression in SBC-5 cells using the intrakine strategy and evaluated the biological behavior of these cells, including proliferation, apoptosis, cell cycle progression, invasion and migration in vitro. In addition, we used the multiple organ metastasis model of human SCLC cells to study organ metastasis in vivo. Our results revealed that CXCR4 was a candidate gene involved in metastasis to specific organs, such as the lungs, liver and bone. However, the silencing of CXCR4 did not affect the proliferation and apoptosis of the SBC-5 cells in vitro. CXCR4 may prove to be a promising target for the prevention and effective treatment of metastastic lesions due to SCLC.

\section{Materials and methods}

Cell culture. The human SCLC cell lines, SBC-5 and SBC-3, were gifts from Professor Saburo Sone and Professor Seiji Yano (Tokushima University, Tokushima, Japan). They were maintained at $37^{\circ} \mathrm{C}$ with $5 \% \mathrm{CO}_{2}$ in RPMI-1640 supplemented with $10 \%(\mathrm{v} / \mathrm{v})$ heated-inactivated fetal bovine serum (FBS; both from Gibco, Gaithersburg, MD, USA), $100 \mathrm{U} / \mathrm{ml}$ streptomycin and $100 \mathrm{U} / \mathrm{ml}$ penicillin.

Flow cytometric analysis of CXCR4 expression in SBC-5 and $S B C-3$ cells. SBC-5 and SBC-3 cells (or stably transfected SBC-5/S-K and SBC-5/neo cells) were collected and washed with phosphate-buffered saline (PBS) supplemented with $0.5 \%$ BSA. The cells were then resuspended to a final concentration of $4 \times 10^{6}$ cells $/ \mathrm{ml}, 25 \mu \mathrm{l}$ of which were extracted for staining. In brief, this was followed by the addition of $10 \mu \mathrm{l}$ carboxyfluorescein-conjugated mouse anti-human CXCR4 (clone 12G5) monoclonal antibodies (FAB170F; R\&D Systems, Minneapolis, MN, USA) and incubation for $30 \mathrm{~min}$ at $4^{\circ} \mathrm{C}$. Subsequently, the cells were washed with PBS to remove the unreacted antibodies and were then resuspended in 200-400 $\mu \mathrm{l}$ of PBS. The cell surface expression of CXCR4 was measured using a flow cytometer (BD Biosciences, San Jose, CA, USA) using a $488 \mathrm{~nm}$ wavelength laser excitation. The cells expressing CXCR4 were fluorescently stained, with the intensity of staining directly representing the density of CXCR4. The negative controls cells were stained with PBS.

Construction and use of recombinant plasmid, PCMV-S-K. The recombinant plasmid, PCMV-S-K, was gift from Professor Ping Zhong Wang (Center of Diagnosis and Treatment for Infectious Diseases, Tangdu Hospital, the Fourth Military Medical University, Xi'an, China). It was transfected into competent Escherichia coli DH5 $\alpha$ cells, and then cultured in LB agar plates to select colonies with inserted SDF-1-KDEL sequences using colony polymerase chain reaction (PCR) with primers (sense, 5'-CACCATGAACGCCAAGGTC-3', antisense, 5'-CAGCTCGTCCTTTTACTTGTTT-3'). Colonies with an inserted sequence were identified by agarose gel electrophoresis. The sequence of the plasmid correctly inserted with SDF-1-KDEL was verified by DNA sequencing.
Stable transfection. The PCMV-S-K and the PCMV mock vector were transfected into the SBC-5 cells using Lipofectamine 2000 (Invitrogen, Carlsbad, CA, USA) following the manufacturer's instructions. Plasmid GFP was transfected at the same time as positive control to show its transfection efficiency. The transfected cells were selected with medium containing $100 \mu \mathrm{g} / \mathrm{ml}$ of G418 sulfate (Geneticin; Invitrogen) from the second day $(100 \mu \mathrm{g} / \mathrm{ml}$ is the minimum concentration of geneticin which can kill all SBC-5 cells within 2 weeks). After 1 month of transfection, G418-resistant colonies were isolated by limiting dilution and then expanded. The stably transfected cells were designated as the SBC-5/S-K and SBC-5/neo cells, respectively and maintained in growth mediumcontaining $50 \mu \mathrm{g} / \mathrm{ml}$ of Geneticin.

Detection of SDF-1 expression by immunofluorescence. The SBC-5/S-K and SBC-5/neo cells in the logarithmic growth phase were collected, washed, fixed and incubated for $5 \mathrm{~min}$ at room temperature. They were then washed for $15 \mathrm{~min}$ with PBS containing $0.5 \%$ Triton $\mathrm{X}-100$, and incubated for $1 \mathrm{~h}$ at $37^{\circ} \mathrm{C}$ in blocking solution. The rabbit-anti-human SDF-1 polyclonal antibody (FL-93, sc-28876; 1:50 dilution; Santa Cruz Biotechnology, Santa Cruz, CA, USA) was added followed by incubation for $2 \mathrm{~h}$ at $37^{\circ} \mathrm{C}$; the cells were then washed and incubated in fluorescein isothiocyanate (FITC)-conjugated goat-anti-rabbit IgG antibody (H+L, bsF-0295G; Bioss, Woburn, MA, USA; 1:100 dilution with PBS containing $0.01 \%$ Evans blue) at $37^{\circ} \mathrm{C}$ for $1 \mathrm{~h}$. Finally, the cells were washed and coverslips were mounted in $50 \%$ buffered glycerol mounting solution. Microscopic images to detect the expression of SDF-1 were obtained using an Olympus BX51 inverted fluorescence microscope (Olympus, Tokyo, Japan). For the negative controls, the process was carried out by substituting the primary antibody with PBS.

In vitro cell proliferation assay. To measure the cell proliferation of the SBC-5/S-K and SBC-5/neo cells, cells at $80 \%$ confluence were harvested and placed into 96 -well plates (1,000 cells/well). Each day 1 plate was used for MTT assay. A total of $20 \mu \mathrm{l}$ MTT solution $(5 \mathrm{mg} / \mathrm{ml})$ was added to each well followed by incubation at $37^{\circ} \mathrm{C}$ for $4 \mathrm{~h}$. Subsequently, the MTT solution was removed and $150 \mu \mathrm{l}$ of dimethyl sulfoxide (DMSO) were added to dissolve the formazan crystals. Absorbance was detected at reference wavelengths of 490 and $630 \mathrm{~nm}$ using an ELISA plate reader (Multiskan MK3; Thermo Fisher Scientific, Inc., Waltham, MA, USA). This assay was performed 3 times.

Soft agar colony formation assay. Twenty-four-well plates were covered with $0.6 \%$ agar in RPMI-1640 medium containing $10 \%$ FBS to prevent the attachment of the cells to the plastic substratum. Cell suspensions $\left(1 \times 10^{3}\right.$ cells/well) of the SBC-5/S-K and SBC-5/neo cells with $0.3 \%$ agar were prepared and seeded on the foundation agar. After 2 weeks of incubation at $37^{\circ} \mathrm{C}$, the colonies containing at least 50 cells were counted under an inverted microscope (IX53; Olympus). All assays were performed 3 times.

Cell apoptosis and cell cycle analysis. Cell apoptosis was determined by flow cytometry using the Annexin V-FITC apoptosis detection kit (Calbiochem, La Jolla, CA, USA). Cells $\left(1 \times 10^{6}\right)$ were collected and washed twice with cool PBS. The cells 
were then resuspended in $1 \mathrm{X}$ binding buffer. Annexin V-FITC and PI were then added and the cells were incubated at room temperature for $15 \mathrm{~min}$ in the dark. The cells were again washed with cool PBS twice. Finally, $500 \mu 1$ PBS were added to the mixture which was analyzed using a FACSCalibur flow cytometer (BD Biosciences, San Jose, CA, USA).

Cell cycle distribution was determined by the following steps: the cells were resuspended at $1 \times 10^{6}$ cells $/ \mathrm{ml}$, fixed with $75 \%$ ethanol at $4{ }^{\circ} \mathrm{C}$ overnight, washed and resuspended with cool PBS. Subsequently, $5 \mu 1 \mathrm{RNase}(10 \mathrm{mg} / \mathrm{ml})$ were added, and the cells were fixed for $1 \mathrm{~h}$ at $37^{\circ} \mathrm{C} ; 100 \mu \mathrm{g} / \mathrm{ml}$ propidium iodide in a $0.1 \%$ sodium citrate $/ 0.1 \%$ Triton X-100 solution was then added, followed by incubation for $30 \mathrm{~min}$ at room temperature in the dark. After the cells were washed, the analysis of cellular DNA content was carried out using a flow cytometer (BD Biosciences, San Jose, CA, USA) at an excitation wavelength of $488 \mathrm{~nm}$. The distribution of these cells in 3 major phases of the cell cycle (G1, S and G2 phase) was analyzed using CellQuest and ModFit software (BD Biosciences, San Jose, CA, USA).

In vitro migration and invision assay. To detect cell migration in vitro, 24-well Transwell plates containing filters of $8.0 \mu \mathrm{m}$ pore size (Costar, Cambridge, MA, USA; Matrigel uncoated) were used. The SBC-5/S-K and SBC-5/neo cells $\left(5 \times 10^{4}\right)$ in RPMI-1640 medium containing 0.1\% BSA (Sigma-Aldrich Co., St. Louis, MO, USA) were placed into the upper chamber of the wells and RPMI-1640 containing 10\% FBS was added to the lower chamber. The Transwell plates were incubated for $36 \mathrm{~h}$. The filters were fixed with $10 \%$ formalin, and stained with crystal violet. The cells on the upper surface of the filters were removed by swabbing with a cotton swab and the cells migrated to the lower surface were counted under a microscope (IX53; Olympus) at x200 magnification. Ten fields for each sample were counted and analyzed. All assays were performed 3 times.

Cell invasion assays were performed using Matrigel-coated (BD Biosciences, Le Pont-de-Claix, France) 24-well Transwell plates. The other steps were the same as those used for the migration assay.

Determination of metastasis in vivo. All animal experiments were performed according to the Guidelines on Animal Experimentation formulated by the Forth Military Medical University, Xi'an, China. The SBC-5/S-K and SBC-5/neo cells were harvested and only a single cell suspension with $>90 \%$ cell viability were used. NOD-SCID mice at 3-4 weeks of age (from the Institute of Biochemistry and Cell Biology of Shanghai Institute for Biological Sciences, Chinese Academy of Sciences, China) were divided into 2 groups and each group consisted of 2 male and 3 female mice. The SBC-5/S-K and SBC-5/neo cells $\left(1 \times 10^{6} / 300 \mu \mathrm{l}\right)$ were injected into the tail vein of the mice, which were maintained under specific pathogen-free conditions. After 5 weeks, the mice were anesthetized and bone metastases were visualized by X-ray images. The number of osteolytic bone metastases on the X-ray images was evaluated independently by 2 investigators (Dr Haichuan Su and Professor Minzhang Tang, Department of Oncology, Tangdu Hospital, The Fourth Military Medical University, Xi'an, China).

Subsequently, the mice were sacrificed by anesthesia and all the major organs were removed. The number of metastatic lesions $>0.5 \mathrm{~mm}$ in diameter on the surface of the major organs was counted macroscopically. The lungs were fixed in Bouin's solution for $24 \mathrm{~h}$. The major organs with metastastic lesions were fixed in 10\% formalin. The bone specimens were decalcified in $10 \%$ EDTA solution for 1 week and then embedded in paraffin.

Statistical analysis. The Wilcoxon rank sum test was used to determine the significance of the differences in the number and incidence of metastatic lesions in multiple organs/tissues (bone, lungs, liver and kidneys) between 2 groups, and the other data were analyzed by variance analyis or the t-test. Statistical tests were performed using SPSS software version 13.0.0 (SPSS Inc., Chicago, IL, USA). A value of $\mathrm{P}<0.05$ was considered to indicate a statistically significant difference and all statistical tests performed were two-sided.

\section{Results}

Expression levels of CXCR4 in SBC-5 cells are higher than those in SBC-3 cells. We determined the expression levels of CXCR4 in the SBC-5 and SBC-3 cells by flow cytometric analysis and found that the CXCR4 expression rate was $53.04 \pm 1.35 \%$ in the SBC- 5 cells and $25.91 \pm 0.78 \%$ in the SBC- 3 cells $(\mathrm{P}=0.000002)$ (Fig. 1). This assay indicated that the surface expression of CXCR4 in the SBC-5 cells was markedly higher than that in the SBC-3 cells.

Expression of the recombinant fusion protein SDF-KDEL in $S B-5 / S-K$ cells. After we confirmed the recombinant plasmid PCMV-S-K by colony PCR and DNA sequencing (Fig. 2), the PCMV-S-K vector and the PCMV mock vector were transfected into the SBC-5 cells and the stably transfected cells were designated as SBC-5/S-K and SBC-5/neo cells, respectively. Of these cells, $80 \%$ were GFP-positive within $24 \mathrm{~h}$ after transfection (Fig. 3A). We examined the location of the recombinant fusion protein, SDF-KDEL, in the SBC-5/S-K and SBC-5/ neo cells by immunofluorescence staining. SDF-1 was mainly expressed in the cytoplasm of the SBC-5/S-K cells, particularly in the perinuclear region (Fig. 3C). However, the expression of SDF-1 was not detected in the SBC-5/neo cells (Fig. 3B).

Downregulation of CXCR4 in SBC-5/S-K cells compared with $S B C-5 /$ neo cells. To examine the biological activity of the produced SDF-KDEL fusion protein, the expression of CXCR4 in the SBC-5/S-K cells was monitored by flow cytometry. The CXCR4 expression rates in the SBC-5/S-K and SBC-5/neo cells were $10.08 \pm 1.49$ and $50.50 \pm 2.31 \%$, respectively ( $\mathrm{P}=0.000006)$ (Fig. 4). These results indicated that the cells transfected with the recombinant plasmid, pCMV-S-K, presented with a significantly reduced expression of CXCR4 on the cell surface.

Downregulation of CXCR4 has no effect on cell proliferation. Subsequently, the effects on cell proliferation of the downregulation of CXCR4 in the SBC-5 cells were analyzed by MTT assay and soft agar colony formation assay. From the cell proliferation curve, we concluded that the proliferation of the SBC-5/S-K cells did not differ significantly from that of the SBC-5/neo cells (Fig. 5A). Furthermore, the colonies formed by these 2 types of cells also showed no differences (210.75 \pm 10.89 and $226.25 \pm 13.31, \mathrm{P}=0.4$; Fig. 5B). These results 

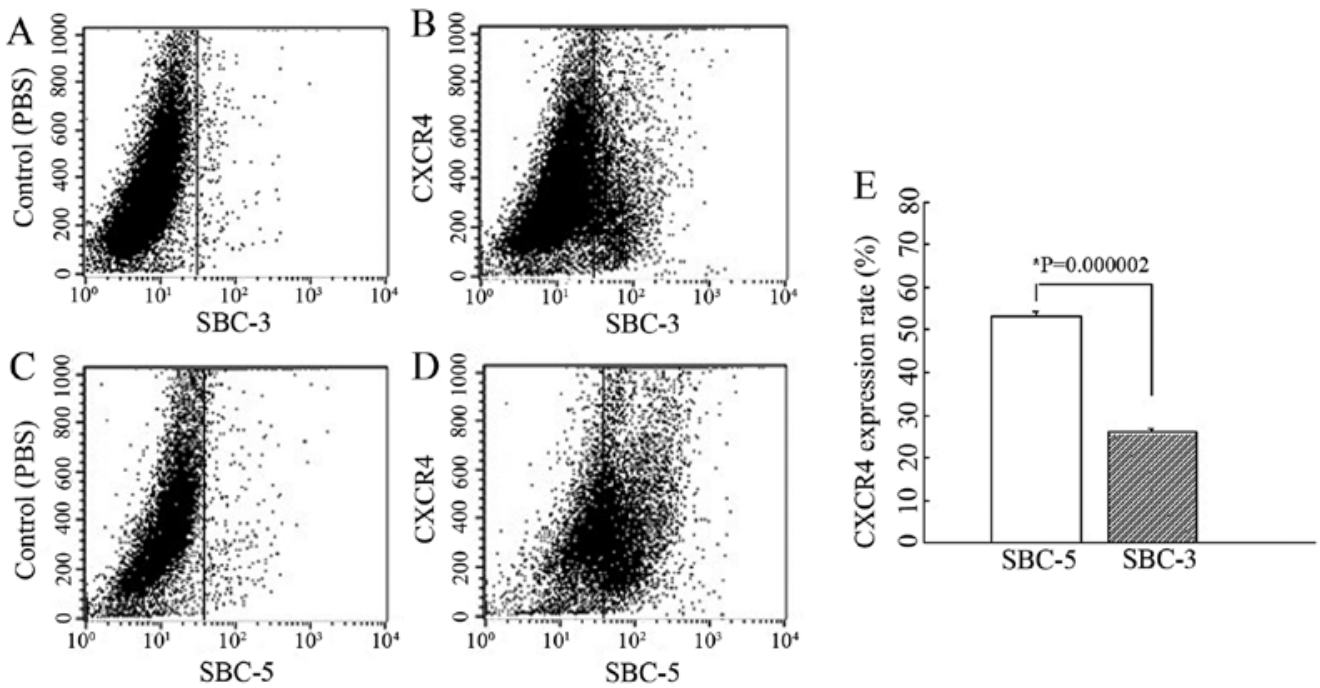

Figure 1. Differential expression of C-X-C chemokine receptor type 4 (CXCR4) in the human small cell lung cancer cell lines, SBC-5 and SBC-3. (A-D) Representative graphs of flow cytometric analysis of CXCR4 expression in (A and B) SBC-3 cells and (C and D) SBC-5 cells. (E) Results from statistical analysis are presented as the means $\pm \mathrm{SD}\left({ }^{*} \mathrm{P}=0.000002\right)$.

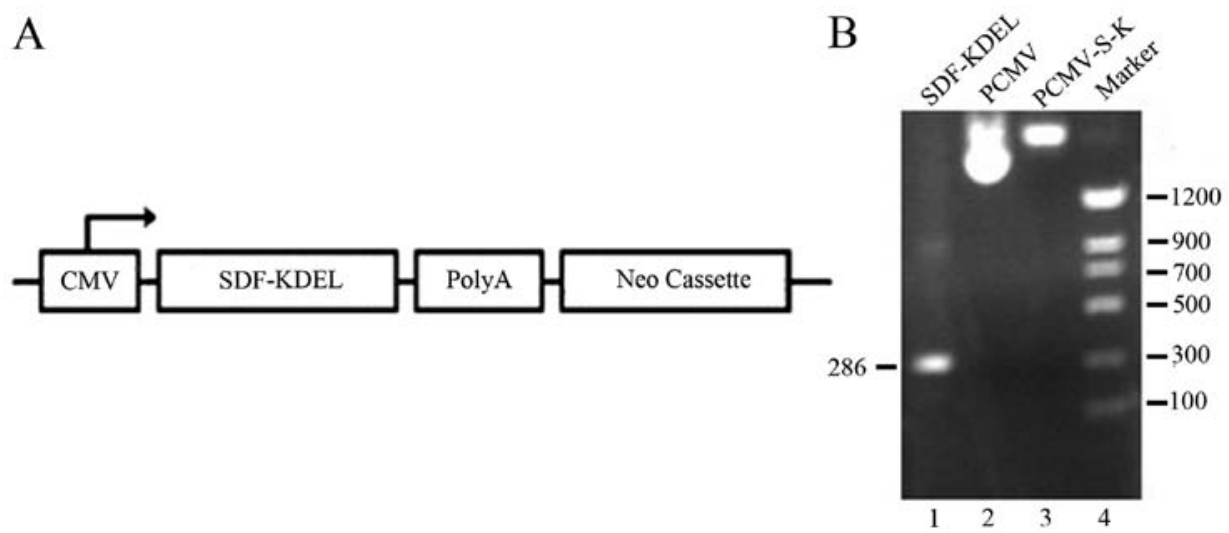

Figure 2. Construction of the recombinant plasmid, PCMV-S-K. (A) Sketch of the recombinant plasmid, PCMV-S-K. (B) Agarose gel electrophoresis of the recombinant plasmid, PCMV-S-K, colony PCR and PCMV mock vector. Lane 1, colony with a 286 bp band, representing the colony with the anticipated oligomer SDF-1-KDEL; lane 2, pCMV mock vector; lane 3, recombinant plasmid PCMV-S-K fragment on a 1\% agarose gel; lane 4, marker.
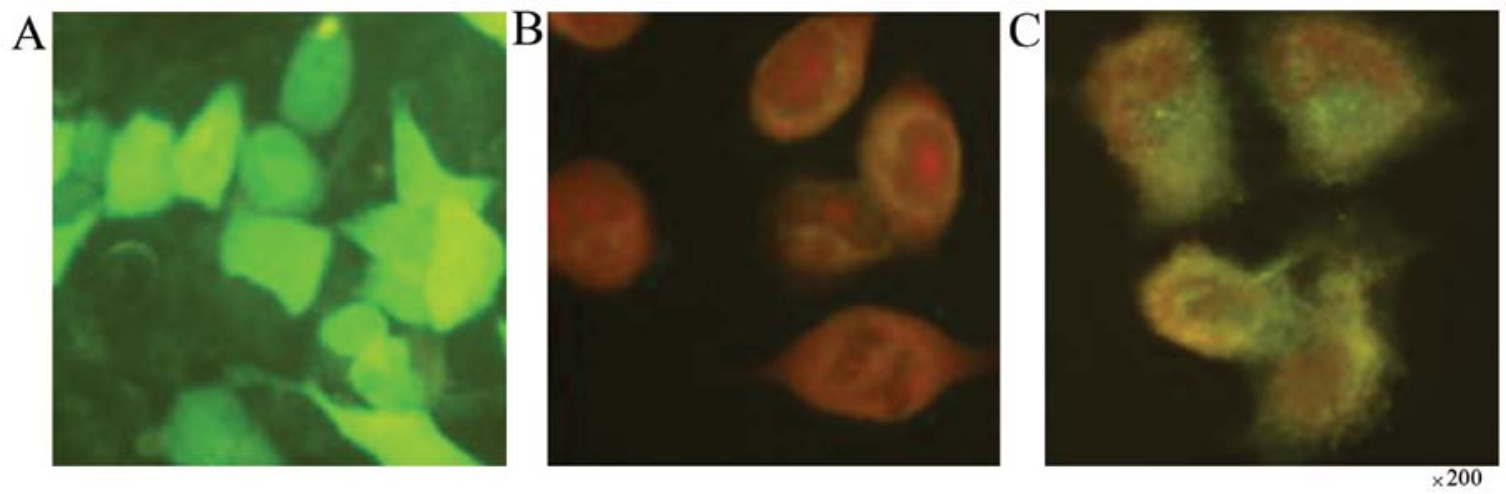

Figure 3. Expression of the recombinant fusion protein, SDF-KDEL, in the SBC-5 cells transfected with the PCMV-S-K vector (SBC-5/S-K cells). (A) GFP expression on the second day after transient transfection of the GFP plasmid into SBC-5 cells, representing the transfection efficiency. (B) Expression of the recombinant fusion protein, SDF-KDEL, in SBC-5/neo cells as a negative control. (C) Expression of the recombinant fusion protein, SDF-KDEL, in SBC-5/S-K cells.

suggested that the downregulation of CXCR4 had no effect on the proliferation of the SBC-5 cells.
Downregulation of CXCR4 does not affect the apoptotic rate of SBC-5 cells. The analysis of cell apoptosis revealed that the 

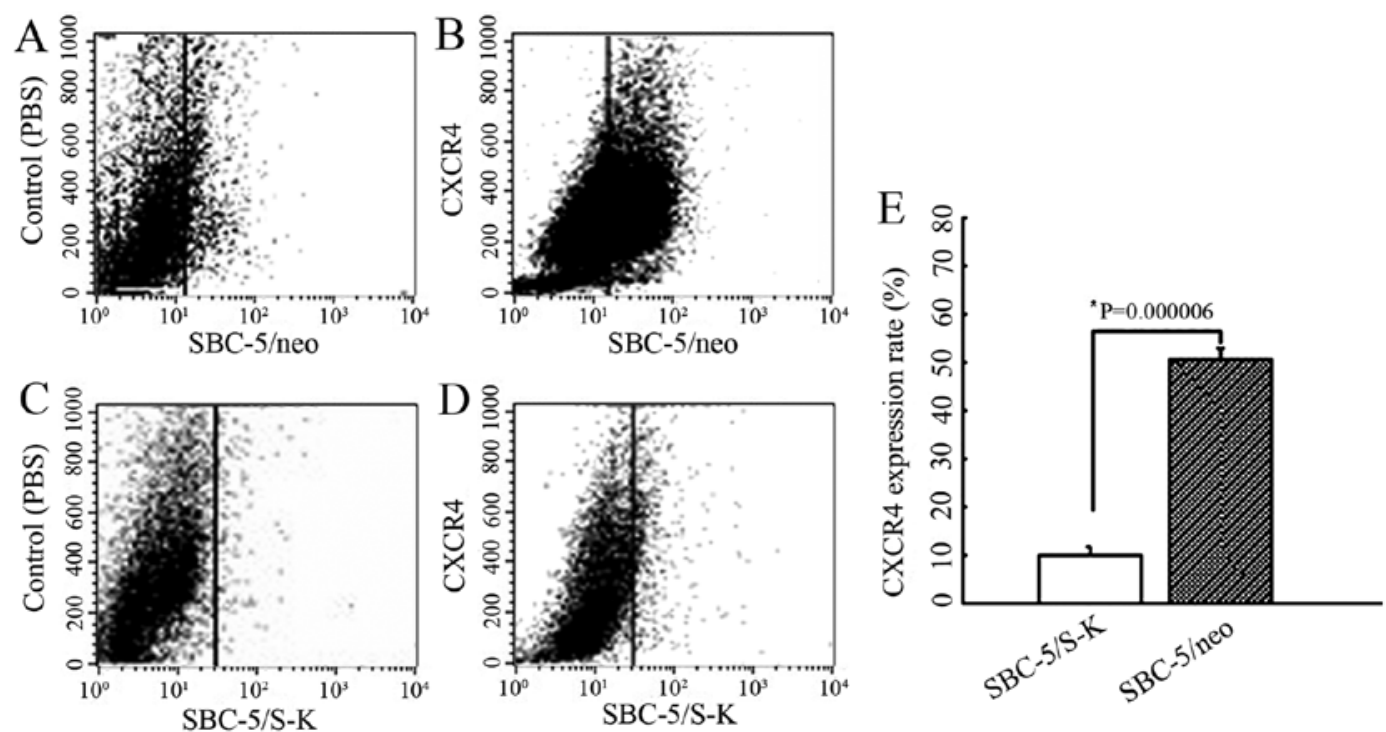

Figure 4. Downregulation of C-X-C chemokine receptor type 4 (CXCR4) in SBC-5 cells transfected with the PCMV-S-K vector (SBC-5/S-K cells) compared to SBC-5/neo cells control cells transfected with PCMV mock vector. (A-D) Representative graphs of flow cytometric analysis of CXCR4 expression in (A and B) SBC-5/neo cells and (C and D) SBC-5/S-K cells. (E) The results from statistical analysis are presented as the means \pm SD (" $\mathrm{P}=0.000006$ ).
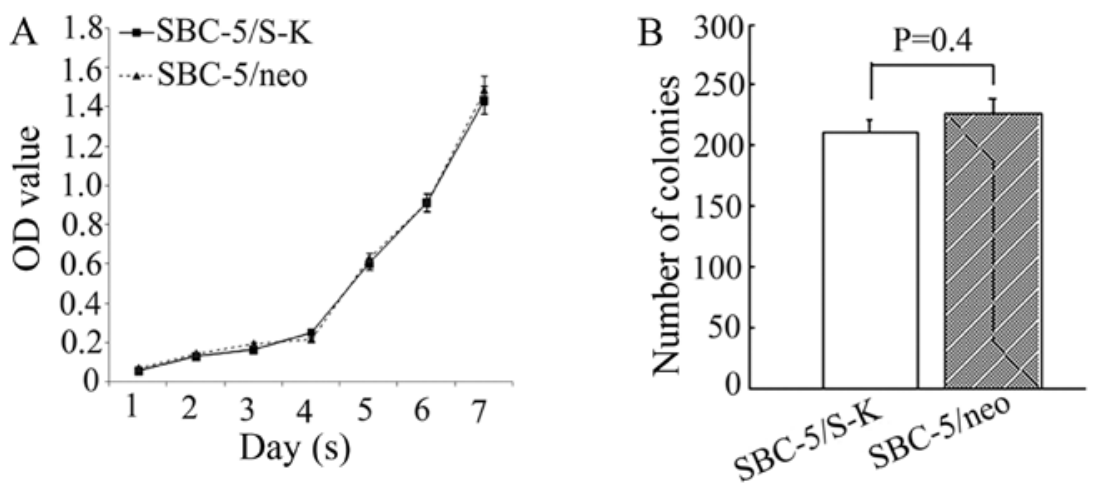

Figure 5. Effect of the downregulation of C-X-C chemokine receptor type 4 (CXCR4) expression on the proliferation and colony formation of SBC-5 cells. (A) Cell proliferation curve of SBC-5/S-K and SBC-5/neo cells ( $\mathrm{P}>0.05)$. (B) Number of colonies formed by SBC-5/S-K and SBC-5/neo cells displayed as the means $\pm \mathrm{SD}(\mathrm{P}=0.40)$.

A

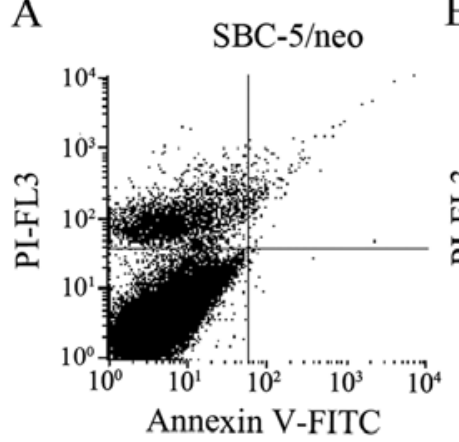

B

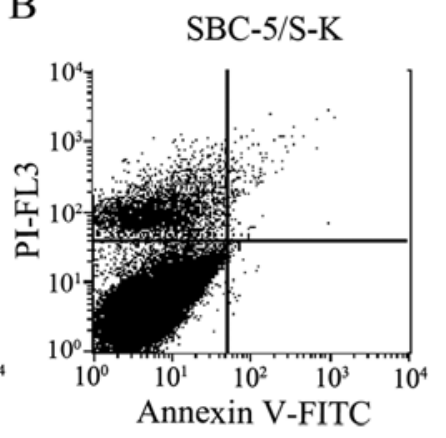

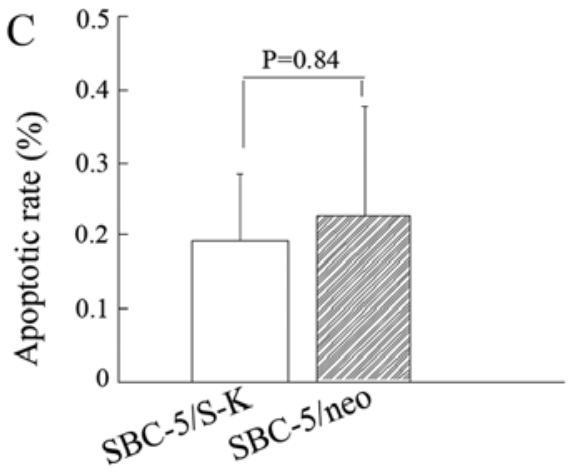

Figure 6. Cell apoptosis was analyzed by FACS analysis. (A) Representative image of cell apoptotic rate of SBC-5/neo cells. (B) Representative image of cell apoptotic rate of SBC-5/S-K cells. (C) Apoptotic rates of the SBC-5/neo and SBC-5/S-K cells are presented as the means \pm SD. (P>0.05).

apoptotic rates of the SBC-5/S-K and SBC-5/neo cells were $0.19 \pm 0.0967$ and $0.2275 \pm 0.15 \%$, respectively ( $\mathrm{P}=0.84$; Fig. 6).
This assay indicated that the downregulation of CXCR4 did not affect the apoptotic potential of the SBC-5 cells. 

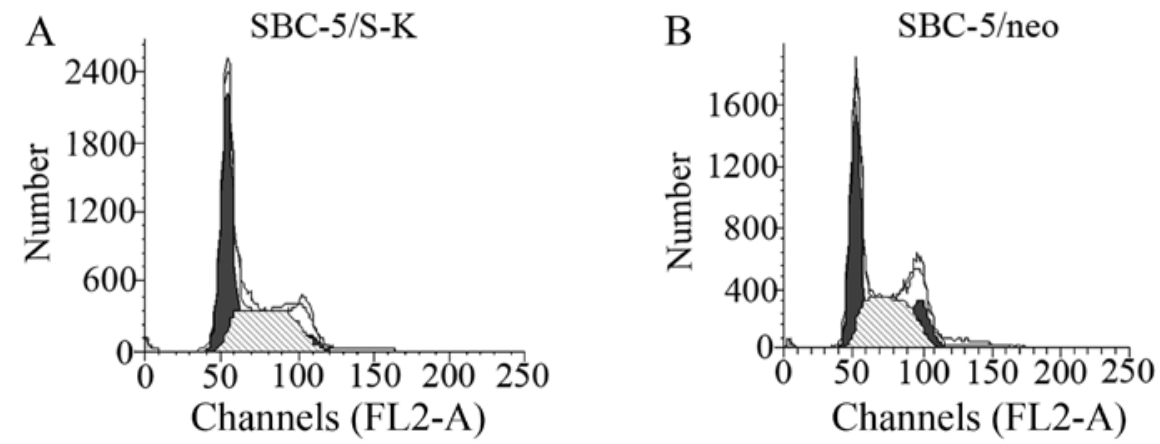

Figure 7. The cell cycle was analyzed by FACS. The distribution of cell cycle phases in (A) SBC-5/S-K cells and (B) SBC-5/neo cells.

A

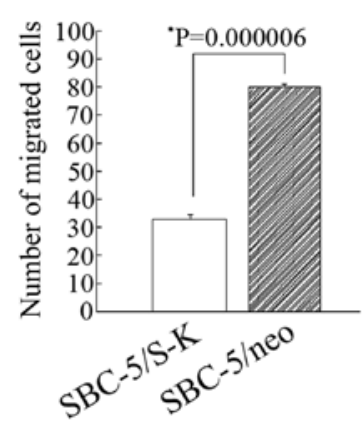

$\mathrm{B}$

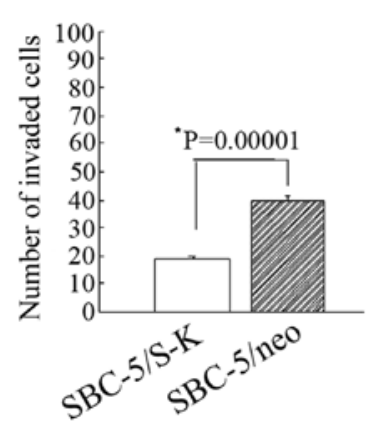

Figure 8. Migration and invasion assay. (A) Mean number of SBC-5/S-K and SBC-5/neo cells that had migrated across the Transwell membranes ( $\mathrm{P}=0.000006)$. (B) Mean number of SBC-5/S-K and SBC-5/neo cells that had invaded the Matrigel-coated Transwell membranes ( $\mathrm{P}=0.00001)$. Bars represent the means \pm SD.

Table I. Cell cycle distribution of SBC-5/S-K and SBC-5/neo cells.

\begin{tabular}{lrrr}
\hline & \multicolumn{3}{c}{ Cell cycle phase } \\
\cline { 2 - 4 } Cell group & \multicolumn{1}{c}{ G1 (\%) } & \multicolumn{1}{c}{$\mathrm{S}(\%)$} & \multicolumn{1}{c}{ G2 (\%) } \\
\hline SBC-5/S-K & $49.64 \pm 5.65$ & $46.7 \pm 6.50$ & $5.32 \pm 2.13$ \\
SBC-5/neo & $44.775 \pm 2.21$ & $50.49 \pm 1.32$ & $4.73 \pm 1.58$ \\
\hline
\end{tabular}

The cell cycle was analyzed by propidium iodide staining and flow cytometry. Distribution of the cell cycle phases in SBC-5/S-K and $\mathrm{SBC}-5 /$ neo cells are shown as the means \pm standard deviation $(\mathrm{SD})$.

Downregulation of CXCR4 does not affect the cell cycle distribution of SBC-5 cells. The percentage of SBC-5/S-K and SBC-5/neo cells in the G1 phase of the cell cycle of was $49.64 \pm 5.65$ and $44.775 \pm 2.21 \%$, respectively $(\mathrm{P}=0.44)$, and that in the $\mathrm{S}$ phase was $46.7 \pm 6.50$ and $50.49 \pm 1.32 \%$, respectively $(\mathrm{P}=0.6)$ (Fig. 7 and Table I). These result indicated that the downregulation of CXCR4 did not affect the cell cycle distribution of SBC-5 cells.

Downregulation of CXCR4 inhibits the migration and invasion of $S B C-5$ cells. Through preliminary experiments, we found that there was a marked difference in the number of SBC-5/S-K and SBC-5/neo cells that had migrated through the insert $(33 \pm 1.73$ and 80.2 $\pm 4.2, \mathrm{P}=0.000006$; Fig. $8 \mathrm{~A}$ ). The number of invaded SBC-5/S-K and SBC-5/neo cells was $19.2 \pm 0.86$ and $39.6 \pm 1.96$, respectively ( $\mathrm{P}=0.00001$; Fig. 8B). These results indicated that the downregulation of CXCR4 significantly decreased the invasion and migration capability of the SBC-5 cells.

Downregulation of CXCR4 inhibits metastasis in vivo. Finally, we examined the role played by CXCR4 in vivo. At the 5th week after inoculation, paralysis (possibly caused by spinal cord compression and bone metastases in the hind limbs) occurred in $1 / 5$ mice in the SBC-5/S-K group and in 5/5 mice in the SBC-5/ neo group. In addition, bone metastasis occurred in $3 / 5$ mice in the SBC-5/S-K group, but in $100 \%$ of the mice in the SBC-5/neo group (Table II). The number of metastatic lesions observed in the bone, liver and lungs in the SBC-5/S-K group was significantly decreased compared with that in the SBC-5/neo group $(\mathrm{P}=0.01$; Table II); however, the number of metastatic lesions observed in the kidneys did not differ significantly the 2 groups $(\mathrm{P}>0.05)$. These results revealed that the downregulation of CXCR4 significantly inhibited specific metastasis to the bone, liver and lungs. It can thus be hypothesized that SDF-1-CXCR4 is involved in the organ-selective metastasis to the lungs, liver and bone. The images of bone metastatic lesions are shown in Fig. 9.

\section{Discusion}

Chemokines are a large family of small, structurally related heparin-binding proteins classified as XCL, CXCL, CCL and CX3CL1 subfamilies depending on the number and spacing of conserved cysteine residues near the $\mathrm{N}$-terminus. Chemokines interact with seven-transmembrane $\mathrm{G}$ protein-coupled chemokine receptors. More than 40 chemokines and 18 chemokine receptors have been discovered, and some chemokines bind to 
Table II. Metastatic lesions formed in NOD-SCID mice following inoculation with SBC-5/S-K and SBC-5/neo cells for 5 weeks.

\begin{tabular}{|c|c|c|c|c|c|c|c|c|}
\hline \multirow[b]{2}{*}{ Cell type } & \multicolumn{2}{|c|}{ Bone } & \multicolumn{2}{|c|}{ Lung } & \multicolumn{2}{|c|}{ Liver } & \multicolumn{2}{|c|}{ Kidney } \\
\hline & Incidence & $\mathrm{N}$ & Incidence & $\mathrm{N}$ & Incidence & $\mathrm{N}$ & Incidence & $\mathrm{N}$ \\
\hline SBC-5/S-K & $3 / 5$ & $1(0-3)^{\mathrm{a}}$ & $5 / 5$ & $3(2-5)^{\mathrm{a}}$ & $5 / 5$ & $3(2-4)^{a}$ & $5 / 5$ & $5(4-6)$ \\
\hline SBC-5/neo & $5 / 5$ & $5(4-6)$ & $5 / 5$ & $8(7-9)$ & $5 / 5$ & $8(7-11)$ & $5 / 5$ & $6(3-7)$ \\
\hline
\end{tabular}

The incidence of metastases in mice and the number of metastatic lesions in bone, lungs, liver and kidneys at the 5th week after inoculation of the 2 groups of cells. Values are shown as the means $\pm \mathrm{SD}$. ${ }^{\mathrm{a} P}=0.01$ compared with the control group (Wilcoxon rank sum test).

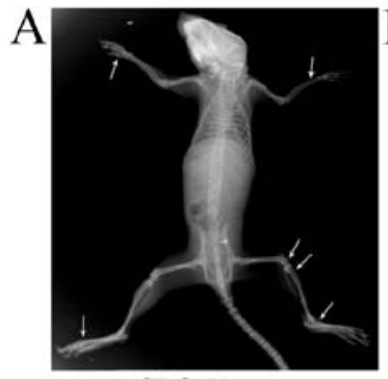

$\mathrm{SBC}-5 /$ neo

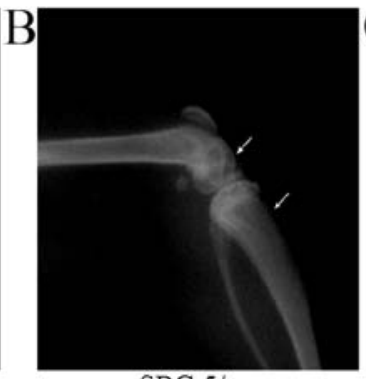

SBC-5/neo

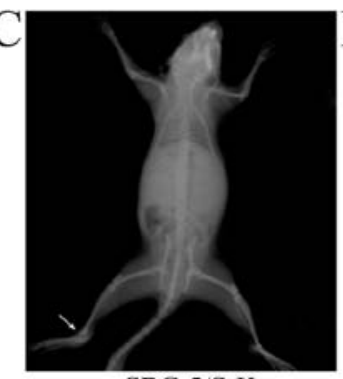

SBC-5/S-K

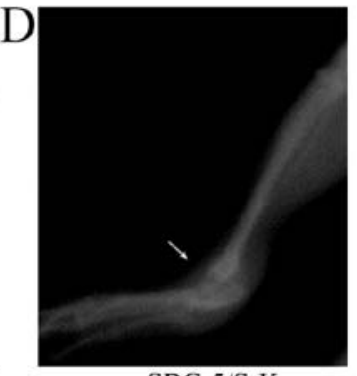

SBC-5/S-K

Figure 9. X-ray images of osteolytic bone metastases formed following inoculation of the cancer cells for 5 weeks. (A and B) Bone metastatic lesions in the $\mathrm{SBC}-5 /$ neo group; (C and D) bone metastatic lesions in SBC-5/S-K group.

multiple chemokine receptors or vice versa (12). Chemokines were noted initially for their ability to stimulate the directional migration of nearly all classes of leukocytes (13). Recent evidence indicates that members of the chemokines and their receptors may play critical roles in tumorigenesis and/or metastasis (14).

CXCR4 is by far the most common chemokine receptor that has been demonstrated to be overexpressed in a broad array of human cancer tissues, but its expression is low or absent in many normal tissues (15). Its sole ligand, CXCL12, was found mainly in 2 isoforms $\alpha$ and $\beta$, and isoform $\alpha$ is constitutively produced in multiple tissues, including lung, liver and bone tissue (16). There is growing evidence that CXCR4 and SDF-1 regulate migration and metastasis in various types of cancer (17). It has been reported that high levels of CXCR4 expression positively correlate with bone metastasis in breast cancer patients (18). GST-NT21MP, an antagonist of CXCR4 was identified to inhibit the progression of breast cancer (19). Therefore, targeting CXCR4 may be a promising strategy for the treatment of human cancer. The overexpression of CXCR4 in pancreatic cancer, melanoma and neuroblastoma plays an important role in the progression and organ-specific metastasis (20-23). Thus, CXCR4 is considered one of several genes which contribute to bone metastasis in cancer (24). Endothelial cell-derived CXCL12 may trigger the integrin activation to promote the adhesion of cancer cells to the extracellular matrix or accessory cells within the tumor microenvironment. Of note, cancer cells can be retained in the metastatic microenvironment which may confer protection against chemotherapy, and which may be responsible for residual disease and relapses $(25,26)$.

In this study, the experssion of CXCR4 was knocked down using the intrakine strategy with the recombinant plasmid,
PCMV-S-K. It was confirmed that the downregulation of CXCR4 significantly inhibited invasion and migration in vitro and metastasis in vivo. Although the number of metastatic lesions observed in the lungs, bone and liver was decreased in the mice injected with PCMV-S-K cells, metastasis to the kidneys was not suppressed. These results indicated that SDF-1-CXCR4 mediated organ-specific metastasis in SCLC. The mechanisms of action of the SDF-1-CXCR4 axis remain unclear, and thus future studies are required on this issue.

Whether CXCR4 is involved in the survival and proliferation of tumor cells remains controversial, perhaps as this is tumor dependent. The activation of extracellular signal-regulated kinase and Akt can both potentially contribute to the survival and growth of tumor cells $(27,28)$. SDF-1-CXCR4 has been implicated in the organ-specific metastases of many types of cancer, including bone-specific metastasis. The bone marrow is a hypoxic microenvironment and is rich in hypoxia-inducible factor-1 (HIF-1). HIF-1 $\alpha$ and CXCR4 co-operate to regulate the adaptation and survival of cancer- and metastasis-initiating cells (29). In addition, CXCR4 expression can be upregulated by the hypoxia response element, HIF-1 $\alpha(30,31)$. In a relay multistep navigation process, the hypoxia-HIF-1 $\alpha$-CXCR 4 pathway may regulate trafficking in and out of hypoxic tissue microenvironments, which is in favor of cell proliferation, migration, invasion and the formation of tumor/metastases.

Metastases arise following the spread and subsequent growth of cancer cells from a primary site to distant tissues. There are likely to be some special requirements for the formation of metastasis, as evidenced by the existence of a class of genes termed 'metastasis-related genes', which regulate the development of metastatic tumors, but have relatively little effect on the growth of primary lesions. In view of these 
biological functions mediated by CXCR4, it can be concluded that CXCR4 is a gene involved in metastasis in SCLC, and the blockade of the interaction between SDF-1 and CXCR4 may lead to the development of a novel therapeutic strategy for the prevention and treatment of organ-specific metastases in SCLC.

\section{Acknowledgements}

This study was supported by grants from the National Natural Science Foundation of China (nos. 81272345 and 81172011).

\section{References}

1. Robert J: Biology of cancer metastasis. Bull Cancer 100: 333-342, 2013 (In French).

2. Guise T: Examining the metastatic niche: targeting the microenvironment. Semin Oncol 37 (Suppl 2): S2-S14, 2010.

3. Paget S: The distribution of secondary growths in cancer of the breast. 1889. Cancer Metastasis Rev 8: 98-101, 1989.

4. Patel LR, Camacho DF, Shiozawa Y, Pienta KJ and Taichman RS: Mechanisms of cancer cell metastasis to the bone: a multistep process. Future Oncol 7: 1285-1297, 2011.

5. Jemal A, Siegel R, Ward E, Hao Y, Xu J and Thun MJ: Cancer statistics, 2009. CA Cancer J Clin 59: 225-249, 2009.

6. Miki T, Yano S, Hanibuchi M and Sone S: Bone metastasis model with multiorgan dissemination of human small-cell lung cancer (SBC-5) cells in natural killer cell-depleted SCID mice. Oncol Res 12: 209-217, 2000.

7. Ben-Baruch A: Organ selectivity in metastasis: regulation by chemokines and their receptors. Clin Exp Metastasis 25: 345-356, 2008

8. Chen JD, Bai X, Yang AG, Cong Y and Chen SY: Inactivation of HIV-1 chemokine co-receptor CXCR-4 by a novel intrakine strategy. Nat Med 3: 1110-1116, 1997.

9. Bai X, Chen JD, Yang AG, Torti F and Chen SY: Genetic co-inactivation of macrophage- and T-tropic HIV-1 chemokine coreceptors CCR-5 and CXCR-4 by intrakines. Gene Ther 5: 984-994, 1998

10. Zhang JC, Sun L, Nie QH, et al: Down-regulation of CXCR4 expression by SDF-KDEL in CD34(+) hematopoietic stem cells: An anti-human immunodeficiency virus strategy. J Virol Methods 161: 30-37, 2009.

11. Ma WF, Du J, Fu LP, Fang R, Chen HY and Cai SH: Phenotypic knockout of CXCR4 by a novel recombinant protein TAT/54R/ KDEL inhibits tumors metastasis. Mol Cancer Res 7: 1613-1621, 2009.

12. Bachelerie F, Ben-Baruch A, Burkhardt AM, et al: International Union of Basic and Clinical Pharmacology. [corrected] LXXXIX. Update on the extended family of chemokine receptors and introducing a new nomenclature for atypical chemokine receptor. Pharmacol Rev 66: 1-79, 2013.

13. Hayashi H: A review on the natural mediators of inflammatory leucotaxis. Acta Pathol Jpn 32 (Suppl 2): 271-284, 1982.

14. Roy I, Evans DB and Dwinell MB: Chemokines and chemokine receptors: update on utility and challenges for the clinician. Surgery 155: 961-973, 2014.
15. Cojoc M, Peitzsch C, Trautmann F, Polishchuk L, Telegeev GD and Dubrovska A: Emerging targets in cancer management: role of the CXCL12/CXCR4 axis. Onco Targets Ther 6: 1347-1361, 2013.

16. Phillips RJ, Burdick MD, Lutz M, Belperio JA, Keane MP and Strieter RM: The stromal derived factor-1/CXCL12-CXC chemokine receptor 4 biological axis in non-small cell lung cancer metastases. Am J Respir Crit Care Med 167: 1676-1686, 2003.

17. Gebura K and Bogunia-Kubik K: Clinical relevance of chemokine receptor CXCR4. Postepy Hig Med Dosw (Online) 66: 252-266, 2012 (In Polish)

18. Hung CS, Su HY, Liang HH, et al: High-level expression of CXCR4 in breast cancer is associated with early distant and bone metastases. Tumour Biol 35: 1581-1588, 2014.

19. Yang Q, Zhang F, Ding Y, et al: Antitumour activity of the recombination polypeptide GST-NT21MP is mediated by inhibition of CXCR4 pathway in breast cancer. Br J Cancer 110: 1288-1297, 2014.

20. Zhong W, Chen W, Zhang D, et al: CXCL12/CXCR4 axis plays pivotal roles in the organ-specific metastasis of pancreatic adenocarcinoma: A clinical study. Exp Ther Med 4: 363-369, 2012.

21. Toyozawa S, Kaminaka C, Furukawa F, Nakamura Y, Matsunaka $\mathrm{H}$ and Yamamoto Y: Chemokine receptor CXCR4 is a novel marker for the progression of cutaneous malignant melanomas. Acta Histochem Cytochem 45: 293-299, 2012.

22. Sun J, Feng C, Liao W, Zhang H and Tang S: Expression of CXC chemokine receptor- 4 and forkhead box 3 in neuroblastoma cells and response to chemotherapy. Oncol Lett 7: 2083-2088, 2014.

23. Ma M, Ye JY, Deng R, Dee CM and Chan GC: Mesenchymal stromal cells may enhance metastasis of neuroblastoma via SDF-1/CXCR4 and SDF-1/CXCR7 signaling. Cancer Lett 312: $1-10,2011$.

24. Wang J, Loberg R and Taichman RS: The pivotal role of CXCL12 (SDF-1)/CXCR4 axis in bone metastasis. Cancer Metastasis Rev 25: 573-587, 2006.

25. Hartmann TN, Burger JA, Glodek A, Fujii N and Burger M: CXCR4 chemokine receptor and integrin signaling co-operate in mediating adhesion and chemoresistance in small cell lung cancer (SCLC) cells. Oncogene 24: 4462-4471, 2005.

26. Wang B, Wang W, Niu W, et al: SDF-1/CXCR4 axis promotes directional migration of colorectal cancer cells through upregulation of integrin $\alpha v \beta 6$. Carcinogenesis 35: 282-291, 2014.

27. Barbieri F, Bajetto A, Porcile C, et al: CXC receptor and chemokine expression in human meningioma: SDF1/CXCR4 signaling activates ERK1/2 and stimulates meningioma cell proliferation. Ann NY Acad Sci 1090: 332-343, 2006.

28. Wong D and Korz W: Translating an antagonist of chemokine receptor CXCR4: from bench to bedside. Clin Cancer Res 14: 7975-7980, 2008.

29. Mimeault M and Batra SK: Hypoxia-inducing factors as master regulators of stemness properties and altered metabolism of cancer- and metastasis-initiating cells. J Cell Mol Med 17: 30-54, 2013.

30. Sun X, Wei L, Chen Q and Terek RM: CXCR4/SDF1 mediate hypoxia induced chondrosarcoma cell invasion through ERK signaling and increased MMP1 expression. Mol Cancer 9: 17, 2010.

31. Guo M, Cai C, Zhao G, et al: Hypoxia promotes migration and induces CXCR4 expression via HIF-1 $\alpha$ a activation in human osteosarcoma. PLoS One 9: e90518, 2014. 